\title{
Über die Abstammung der Ossa supracleithralia von der Epidermis bei der Forelle.
}

\author{
Von
}

B. Haller.

Hierzu Tafel XxI.

Nachdem Goronowitsch (1) und Julia Platt (5) vorgearbeitet, indem besonders ersterer das Einwandern von Mesenchymzellen vom Ektoderm aus bei Vögeln nachwies, war es bekanntlich Klaatsch (4), der in seiner meiner Ansicht nach höchst wertvollen Schrift den ursprünglichen Bildner des Knochengewebes im Ektoderm, das ist in der Epidermis, erkannte. Kaum war aber diese Schrift veröffentlicht, so trat schon der neuen Lehre C. Rabl (6) auf das Entschiedenste entgegen, indem el Fla atschs Befunde auf einen merklarlichen Irrtum zurïckführen zu müssen meinte.

Damit schien die Sache einschlafen zu wollen, denn obgleich Klaatsch an seinen Befunden festhäl, hat er später die Verteidigung der Lelue nicht mehr aufgenommen. Da dam die gleichlautenden Befunde eines anderen Forschers von ihm selbst widerrufen wurden, so schien die Abstammung des Knochengewebes oder besser des Skelettgewebes von der Epidermis einstweilen beseitigt. Ich habe indessen die Befunde KIatschs für Selachier und Amphibien kontrolliert und mich nicht gescheut, die bestätigten Befunde sogar in ein Lehrbuch (2) aufzunelmen, besonders aber war es die Entwicklung der von mir als Ossa supracleithralia bezeichneten zwei Paal Knochen bei Salmo aus der Epidermis, die ich nebenbei verfolgt hatte (3).

Klatsch nennt diese Anlage die Claviculaanlage und sagt darüber (1. c. S. 202): „Auf Fig. 7 habe ich einen Teil der C'lavicularanlage rom $1,5 \mathrm{~cm}$ langen Salmo salar dargestellt. Wer an solchem Objekt gesehen hat, dass dasselbe nichts anderes darstellt, als eine enorme Wucherung der Epidermis, der wird wohl den letzten Zweifel an der Richtigkeit meiner Amnabme aufgeben. Das Bild spricht für sich, und ich brauche ihm nichts hinzuzufügen." Und doch wäre es gut gewesen, wenn Klatsch gerade 
dies ungemein beweiskräftige Objekt ausführlicher verfolgt und darauf als Beweismaterial das Hauptgewicht gelegt hätte.

Ich habe über diese Ossifikation folgendes berichtet (3): „Es legen sich unterhalb der Seitenlinie gleich hinter dem Opeccularapparat, aus einer epithelialen Einsenkung, zwei lknöcherne Spangen an, die dann nach ventralwärts wachsen bis zu dem primären Schultergürtel. Hier angelangt, kreuzen sie sich und die früher laterale Spange wird zur medianen, erreicht den primären Schulterbogen und legt sich ihm fest an seinem oralwärtigen Rande an. In gleicher Lage wächst diese Spange ventralwärts bis zum Ende des primären Schulterbogens. Indem dann diese Spange die Osteoblasten für den auf dem primüren Schulterbogen sich entfaltenden Coracoid abgibt, entfaltet sich die Spange zum Cleithrum und besitzt das Cleitllium selbst somit nie eine knorpelige Unterlage." I)ann sagte ich: „Es findet eine Unterbrechung der Basalmembran am Boden der Grube (der Epidermisgrube, welche die Anlage der Supracleithralia darstellt) statt, doch erstreckt sich diese Unterbrechung nicht auf den ganzen Bodenteil der Grube, sondern nur anf das Zentrum des Bodens. Dies möchte ich ausdrücklich betonen, denn schon auf dem zweiten oder dritten Schnitte der Serie kam die Membran sich über die Grube hinwegsetzen und fehlt dann auf dem Schnitte die Unterbrechung. . . . Über diesel Jurchblechung tindet eine lebhafte Vermehrung der Epithelzellen der Keimlage statt, obgleich ja diese Lage noch lange nicht so ausgesprochen von den oberen Zellen des Epithels sich ablıebt, wie bei dem entwickelten Tiere. Es entsteht dann als Teilungsprodukt jener Zellen eine grosse Zahl von Skelettoblasten, welche zapfenartig in das Unterbautgewebe hineinragen. Unter dem Zapfen befindet sich das verbreiterte obere Ende der inneren Spange. Der Skelettoblastenzapfen liegt fest der Spange nach aussen an; an den beiden dem Zapfen angrenzenden Ründern aber greifen die Skelettoblasten, im Gefüge locker werdend, auf die innere Seite der Spange über. Sie bilden dann um die Spange eine periostale Hülle und innerhalb der Hülle liegen Osteoblasten, von denen eine Lage der inneren Spangenseite fest anlagert. Die zwischen dieser und dem Perioste gelegenen haben bereits auch spärliche Zwischensubstanz abgeschieden. ... Es bildet dann die Osteoblastanlage eine Scheide um die oberen Enden der Spangen, welche dann nach 
ventralwärts in das anliegende Gewebe allmählich übergeht und dann um das Periost die Spangen nach ventralwirts im Wachstum begleitet."

Diese Angaben bezogen sich auf etwa 3 Wochen alte Tiere von Salmo irideus. Wa ich mir nun \%ur Aufgabe gemacht habe, in vorliegender Schrift diesen Ossifikationsprozess aus der Epidermis ausführlicher zu verfolgen, als dies durch klatsch und mich geschah, um auf diese Weise diese Lehre von der ursprünglichen Abstammung des Knochenskelettes von dem Ektoderm nicht einschlafen zu lassen; so habe ich gleich mit beginnenden $\mathrm{Ku}-$ ständen angefangen.

Bei Embryonen, bei denen das knorpelige Skelett zwar schon fertig, die beiden Supracleithralia aber erst angelegt werden, bildet das Epithel über dieser Anlage keine Grube wie bei jungen Forellen, sondern das Epithel bildet über dieser Anlage einen geringen Hügel, der dann nach allen Seiten sanft abfallt. Auch auf Horizontalschnitten gelangt dieser Hügel noch zum Ausdruck. Es zieht daun die Ossifikationsanlage nach ventralwärts, wie aus lig. 1 auf einem Sagittalschnitte ersichtlich ist. Ich habe durch diese Anlage an den oben genannten Embryonen ein Horizontalschnittserie von sechs Schnitten angefertigt, da ich auf diese Weise weit besser die ersten Anlagen zu erkennen hoftte, als anf Serien einer anderen Schnittrichtung. So war es denn auch. Der dorsalste Schnitt (Fig. 4) zeigt im Epithel gelegen, doch nach der Cutis zu vorgebuchtet, vier $z u$ einem Ring vereinigte \%ellen (b), die auf diese Weise ein Lumen umfassen, das keine irgend welche Füllung besass und darum auch nicht durchtingiert war. Die äusserste der vier Epithelzellèn des Ringes reicht bis an die Obertläche der Epidermis, indessen ihre zwei unteren Fortsätze mit je einer Zelle sich verbinden. Der Ring wird dann auf die Weise der Cutis zu geschlossen, dass eine vierte Zelle mit den zwei oben genannten sich verbindet. Die kaudalwärtigste der vier Zellen unterscheidet sich jetzt schon dadurch von den drei anderrn, dass ihr Zelleib tiefer tingiert ist. Immerhin ist dieser Vierzellenring noch im Epithel gelegen und die Basilarmembran, allerdings sehr verdünnt, schliesst den Ring der Cutis zu ab. Schon der nächstfolgende Schnitt der Serie zeigt die Verhältnisse insofern anders, als der Ring weiter und seine innere Wand mehrschichtig ist und der Ring als solcher schon aus dem Epithel 
in die Cutis herausrückt. I)er zweitfolgende Schnitt zeigt dasselbe in erhöhtem Maße (Fig. 5). Da ist der Ring schon ganz aus dem Epithel (e) in die ('utis gerückt und hängt mit ihm nur durch einen Epithelisthmus zusammen, wobei selbstverständlich die Basilarmembran eben durch jenen Zusammenhang zwischen Epithel und dem Ossifikationsring da unterbrochen ist. Der Epithelring besteht jetzt aus einer äusseren zwei- bis dreischichtigen Lage (a), welche eine innere nur einschichtige Lage (b) umschliesst. Diese innerste einschichtige Lage ihrerseits umfasst noch immer ein leeres Lumen und ihre Zellen unterscheiden sich von jenen der äusseren Ringwand gleich wie die kaudalwärtige Zelle auf Fig. 4 durch eine tiefere Tinktion.

Die nächstfolgenden Schnitte der Serie zeigen dann den ()ssifikationsring ausser Zusammenhang mit dem Epithel, dieses ist dem Ringe gegenüber durch die tieftingierte Basilarmembran alogeschlossell. Dabei ist der ganze Ring dickwandiger, doch die einzellschichtige innere Hälfte des Ringes zeigt noch immer dasselbe wie früher, mit dem Unterschiede, dass das Ringlumen bereits Hartsubstanz ist, bis auf einen dümnen Spalt, der aber weiter nach ventralwärts auch ausgefïllt wird. Dabei sitzt jetzt noch die Hartsubstanz den freien Zellenden jeder Zelle so auf, dass ihre Zugehörigkeit zu je einer Zelle jetzt noch deutlich ist.

Bei einem ilteren Embryo, als der obige, erkannte man auch die Anlage des vorderen Supracleithralknochens, denn die beschriebene bezog sich auf den kaudalwärtigen. Die Anlage ist ganz genau so wie die beschriebene.

Von einem dann noch etwas alteren, schon alten Embryo zeigte sich die ganze Ossifikation in stark vorgeschrittenem Stadium. Davon besitze ich Sagittalschnitte. Noch immer ist die Ossitikationsanlage ein Hügel (Fig. 1). Man sieht die beiden Knochenanlagen, jene des kaudalen und oralen Supracleithrale ( $\mathrm{s}^{\prime}$ ) schon stark vorgeschritten, doch auf den Schnitten immerhin nicht in ganzer Länge. Viel Hartsubstanz liegt schon zwischen den sich verästelnden und mit ihren Fortsätzen untereinander anastomosierenden Ǩnochenzellen, indessen die äusseren Lagen der Osteoblasten mehrschichtig die Knochenanlagen umbüllen (ps). Beide Supracleithralanlagen hängen indessen mit ibrem Mutterboden, dem Epithel (ep) des Hügels (b) durch die geradezu aus dem Epithel a us strömenden Osteoblasten ganz innig zusammen, 
genauestens so, wie ich das schon früher abgebildet labe. Hier auf diesem Schnitte ist dieser Osteoblastenisthmus (z) fïr die beiden Knochenanlagen eine gemeinsame, allein auf den nächstfolgenden Schnitten, auf mehr lateralwätigeren, sieht man noch einen Zusammenhang zwischen der kopfiärtigen Seite des Epithelhügels und der oralen Supracleithralanlage (s), von welchem Zulsammenhang auf dem zu behandelnden Schnitte aber nur noch wenige Zellen sich hier zeigen (b). Dort auf jenem Schnitte wat die Basilarmembran an der angegebenen stelle völig durchbrochen, zeigt hier aber nur noch einige siebfölmige öfinungen, durch die je ein Osteoblast auszutreten vermag. Dies wäre ein nener Befund, denn wie gesagt ist an solchen stellen, wo der Zusammenhang zwischen dem Epithel und der Skelettanlage ein kralftiger ist, die sonst gut tingierte Membran (mb), die "wie mit der Feder ausgezogen" sich reigt, föllig geschwunden. Die anliegenden Teile reigen schon jenes obige Verlatten nicht mehr, doch schliesst sich die Basilarmembran nu allmählich, so dass man auch hied noch kleinere öffinngen erkennt (Fig. 2), durch welche Sklettoblasten (z) in die cutis austreten.

Ich habe danach gesucht ob es demn ausschliesslich die Keimschichte des Epithels ist, wclche skelettoblasten liefert. und tatsächlich macht der auf Fig. I abgebildete Schnitt an der huppe des Hügels jenen Eindruck, als wie wenn dem so wäre. Jedenfalls sind die austretenden Epithelzellen, die Skelettoblasten, sehr nachgiebig und unter Umstanden bei der Wanderung zusammendrückbar, worauf der Unstand ja direkt hinweist, dass an solchen Stellen, an denen Verengung eintritt, Zellkerne der Druckrichtung entsprechend stark zusammengedrückt sein kömmen (Fig. 1 oberhalb bi.

Es zichen ja die Osteoblasten entlang der ganzen Slielettanlage, nach ventralwärts fortwährend die Hartsubstanz abscheidend, soweit sie aber zu Knochenzellen werden. bleiben sie stabil, nur die weiteren Nachschübe machen die Reise mit. Allein nicht alle diese Osteoblasten entstanden in der Epidermis, ein leil sind Tochterkerne der von dort stammenden Osteoblasten selbst und die Zahl solcher sekundärer Bildner darf man keineswegs zu gering schätzen. In allen Fallen sind aber diese, ich möchte sagen. mitreisenden Osteoblasten mehr weniger spindelförmig und nur, wenn sie zu Knochenzellen werden, also stets jene der innersten 
Zellschichte, senden sie Fortsätze aus. Darum sind sie auch, soweit sie lose von aussen der Knochenanlage anlagern, zu unterscheiden von Zellen des embryonalen Bindegewebes (bz), die ja stets verzweigt sind und untereinander anastomosieren.

Ein Querschnitt durch eine ausgeschlüpfte, noch mit Dottersack versehenen Forelle (Fig. 3) zeigt uns die Verhältnisse der Knochenanlagen insofern etwas anders, als der Hügel, wohl infolge des starken Zuges, welche die Supracleithralanlagen nun auf das Epithel infolge ihrer Schwere ausüben, nicht nur verstrichen ist, sondern statt seiner eine wenig tiefe Mulde entsteht, wie ich dies schon früher beschrieben und abgebildet habe. Bei solchen jungen Tierchen sind schon zahlreiche subepitheliale Chromatophoren vorhanden, die stellenweise schon eine Schichte untel der Basilarmembran bilden (pz).

Die Fortsitze solcher Zellen bilden bekanntlich dadurch, dass sie entlang der Basilarmembran hinziehen, unter dieser eine dünne Schichte. Nie findet sich eine solche Schichte zwischen Epithel und dem Osteoblastenisthmus (z) der Skelettanlage, da Osteoblasten und Epithel eben zusammenhangen und an solchen Stellen auch die Basilarmembran durchbrochen ist.

Wie ich schon in meiner zitierten Arbeit mitgeteilt habe, ist die Stelle, an der Skelettanlage $\left(s, s^{\prime}\right)$ und Epithel miteinander zusaminenliangen eine sebr beschrankte. Hinzuzufügen hätte ich noch, dass jene Iustrittsstelle von Skelettoblasten, die beim Embryo oralwärts für die oralwärtige Skelettanlage bestand (Fig. 1 b), bei ausgeschlüptten liorellen sich nicht mehr findet, obgleich der Hauptzusammenhang (Fig. $3 \mathrm{z}$ ) ebenso mächtig ist als ehedem.

Nach diesen Angaben und der Besichtigung der beigegebenen Tafel möchte man aber doch annehmen, dass von nun an diese Ossifikationsstelle sachlich genau geprüft wird, um die Kla atsch sche Lehre neuerdings und mit mehr Ruhe wie bisher zu diskutieren und es nicht darauf ankommen zu lassen, dass die Sache ganz einschlafe und vielleicht nach langen Jahren die Befunde wieder entdeckt würden. So etwas bezeichnet ja immer eine Befangenheit für jene Zeit, in der die Lehre zuerst ausgesprochen ward.

Heute freilich dürtte man ja auch der Auffassung; wonach Mesenchymzellen auch bei den Amnioten aus dem Ektoderm austreten, ohme direkt $z u$ Skelettoblasten $z u$ werden und welche 
452 B. Haller: Über die Abstammung der Ossa supracleithralia etc.

Zustände seinerzeit für die Vögel Groronowitsch geschildert hatte, vertrauensvoller entgegentreten, obgleich scharfe Gegner in grösserer Zahl auch heute nicht fehlen werden. Von Seiten solcher wird aber diese Lehre auch nicht sofort anerkannt werden. Sicher treten Skelettoblasten aber entgegen Kla atsch aus dem Epithel oder Ektoderm bei den Amnioten nicht mehr heraus, sondern bloss Mesenchymzellen, deren Nachkommen oder besser Tochterzellen erst zu solchen werden, denn jener primüre Zustand der direkten Beteiligung des Ectodermes an der Skelettbildung, wie sie Ichthyden und Amplibien heute noch zeigen, hat bei jenen aufgehört.

Heidelberg, im Dezember 1913.

\section{Literaturverzeichnis.}

1. Goronowitsch, N.: Untersuchungen über die Entwicklung der sogrenannten Ganglienleisten etc. Morphol. Jahrb., Bd. XX, 1893.

2. Haller, B.: Lehrbuch der vergleichenden Anatomic. Jeni, 1904.

3. Derselbe: Der Schulterguirtel der Teleostier. Arch. f. milir. Anat., Bd. LXVII, 1905 .

4. Klattsch, H. : Über die Herkunft der Skleroblasten. MLorphol. Jahrb., Bd. XXI, 1894.

5. Platt, J.: Ectodermis Origin of the Cartilages of the Head. Anat. Anz., Bd. VIII, 1893.

6. Rabl, C.: Verhandl. d. Anatom. Gesellschaft. 8. Versammlung, 1894.

\section{Erklärung der Abbildungen auf Tafel XXI.}

Beizieht sich alles auf Salmo irideus. Vergrösserung ${ }^{3}$ s.

Fig. 1. Alter Embryo. Sagittalschnitt. Rei chert. ep=Epithel; h= Hügelkuppe; $\mathrm{mb}=$ Basilarmembran; $z=$ aus dem Epithel austretende Osteoblasten; $\mathrm{s}, \mathrm{s}^{\prime}=$ Skelettanlagen $; \mathrm{bz}=$ Bindegewebszellen; $\mathrm{b}=$ austretende Zellen.

Fig. 2. Sclnitt folgt bald auf den vorigen.

Fig. 3. Junge Forelle mit Dottersack. Querschnitt. $\mathrm{m}=$ Mulde; $\mathrm{pr}=$ Pigmentzellen. Sonst wie zuvor.

Fig. 4. Junger Embryo. Horizontalschnitt. $\mathrm{e}=$ Epithel; $\mathrm{b}=$ Skelettanlage. 


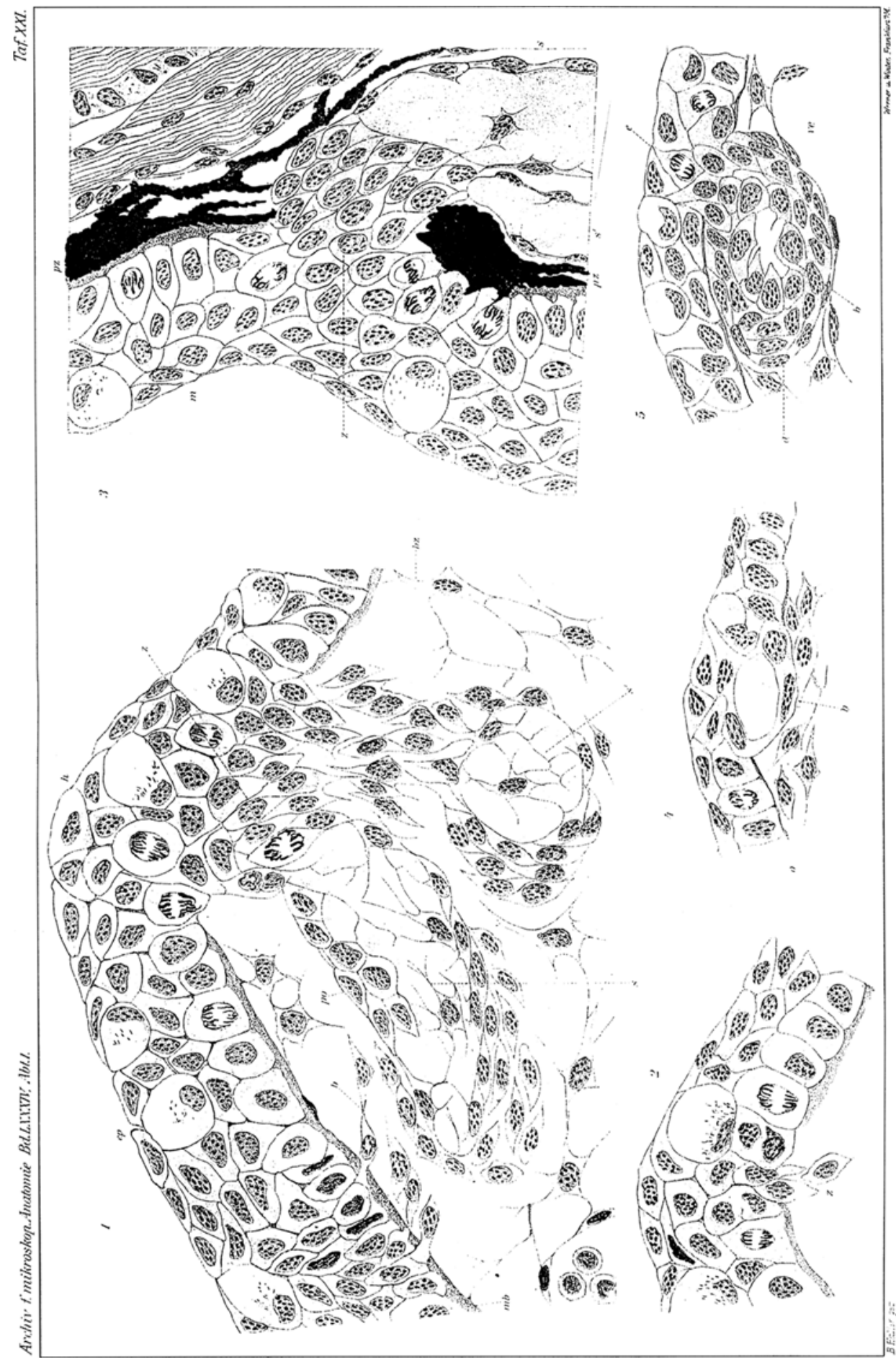

